[17] J. De Villiers and E. Barnard, "Back-propagation neural nets with one and two hidden layers," IEEE Trans. Neural Networks, vol. 4, pp. 135-141, Jan. 1993

[18] V. Phansalkar and P. S. Sastry, "Analysis of the back-propagation algorithm with momentum," IEEE Trans. Neural Networks, vol. 5, pp. 505-507, May 1994.

[19] M. T. Hagan and M. B. Menhaj, "Training feedforward networks with the Marquardt algorithm," IEEE Trans. Neural Networks, vol. 5, pp. 989-993, Nov. 1994.

[20] H. White, "Learning in artificial neural networks: A statistical perspective," Neural Comput., vol. 1, pp. 425-464, 1989.

[21] D. W. Ruck, S. K. Rogers, M. Kabrisky, M. E. Oxley, and B. W. Suter "The multi-layer perceptron as an approximation to a bayes discriminant function," IEEE Trans. Neural Networks, vol. 1, pp. 296-298, Dec. 1990.

[22] M. A. Al-Alaoui, R. Mouci, and M. Mansour, "A redundancy approach to classifier training," in Proc. ICECS, vol. II, Jounieh, Lebanon, Dec. 2000, pp. 950-953.

[23] H. Demuth and M. Beale, Neural Network Toolbox. Natick, MA: The MathWorks, Inc, 1998

[24] R. A. Fisher, "The use of multiple measurements in taxonomic problems," Ann. Eugenics, vol. 7, no. II, pp. 179-188, 1936.

[25] H. Niemann, Pattern Analysis and Understanding. Berlin, Germany: Springer-Verlag, 1989.

[26] R. Mouci, "Neural networks, fuzzy logic and neuro-fuzzy approaches to character recognition," M.S. thesis, Electrical Eng. Dept., American Univ. Beirut, Lebanon, 1997

[27] Iris Data Samples [Online]. Available: ftp.ics.uci.edu/pub/machine-learning databases/iris/iris.data

\section{Comparison Between Different Model of Hexapod Robot in Fault-Tolerant Gait}

Stanley Kwok-Kei Chu and Grantham Kwok-Hung Pang

\begin{abstract}
This paper presents a gait analysis of the equilateral hexagonal model of hexapod robot. Mathematical analysis has been made on mobility, fault-tolerance, and stability. A comparison with the rectangular model of hexapod robot is also given, and it has shown that the hexagonal model shows better turning ability, a higher margin of stability during the fault-tolerant gait, and greater stride length in certain conditions.
\end{abstract}

Index Terms-Hexagonal model, hexapod robot, rectangular model, stability margin.

\section{INTRODUCTION}

One of the fundamental objectives in robotics is to create robots that move around. Many researchers have made comparisons between the use of legs and wheels for locomotion [1], [2]. To summarize, legged robots have the following advantages over wheeled robots:

1) able to traverse on different terrain;

2) higher mobility;

3) lower mechanical coupling between the payload and the terrain;

4) less destructive to the ground.

Manuscript received October 11, 2000; revised September 15, 2001 and September 20, 2001. This paper was recommended by Associate Editor R. A Hess.

S. K.-K. Chu and G. K.-H. Pang are with the Department of Electrical and Electronic Engineering, The University of Hong Kong, Hong Kong (e-mail: gpang@hkueee.hku.hk).

Digital Object Identifier 10.1109/TSMCA.2002.807066
With the above advantages during the past few decades, many legged robots have been built in the laboratory. Depending on design, they have had a number of legs ranging from one to eight. Using a different number of legs involves different methods of control. A six-legged robot, or hexapod, for example, seems to attract more attention than others because it easily achieves static stability during walking. Some researchers [3] believed that the use of six-legged robots would provide a more robust platform for applications, since hexapods can support more weights than bipeds or quadrupeds. Examples of application six-legged robots include, the walking manipulator by Plustech Oy [3], inspection and construction robots, Rosy I and II [4], and Aquarobot for underwater operation [5].

The gait control problem of a hexapod is therefore, an extensively studied subject. McGhee [7] has established many basic terminologies for gait analysis. Bessonov and Umnov [8] have developed hexapod motion in straight lines, and Song and Waldron [1] have made a survey about the gait study. Recently, Yang and Kim [9] have proposed a fault-tolerant gait for a hexapod robot on even terrain. It proved mathematically and graphically, that the gait developed could withstand a fault occurred with reasonable stride length. They later extended the work with an improved fault tolerant gait [10], and another gait over uneven terrain [11].

Among these studies, however, nearly all gaits developed are based upon a simplified rectangular two-dimensional (2-D) model which limits the robot design. Although there are several hexapods (Silex [12], Aquarobot [5], Katharina [13], Odex [14] etc.) built in hexagonal architectures, few analytical comparisons on gait properties have been made between rectangular and hexagonal architectures. Preumont et al. [15] have stated that hexagonal architecture has advantages in turning gait while rectangular has advantages in straight forward gait. No mathematical analysis has been made, howerver. In this paper, we have considered a hexagonal model for gait analysis on the even terrain. In order to show the characteristics of this model, we have made comparisons with the rectangular model in fault tolerant gait. In Section II, some useful definitions, and the traditional rectangular model, will be described first. Section III will describe the new hexagonal model. Comparisons will be made in Section IV, and Section IV will conclude the paper.

\section{PRELIMINARIES}

The common model that represents a hexapod robot for gait analysis is a simplified 2-D model. As shown in Fig. 1, the body of the hexapod is represented by a rectangle. Legs are attached to the longer sides of the rectangle. Leg numbers of the hexapod are 1,2, and 3 on the left-hand side and 4, 5, and 6 on the right-hand side. Each leg has a reachable area in 2-D, or workspace in three-dimensional (3-D). The size and shape of the reachable area depend on the leg design. For this model, the hip joint (i.e., the joint linking the body and leg) is a rotational joint with vertical axis, the leg therefore, having reachable area in the form of an annulus. This kind of leg design, however, arises interference problem as the neighboring legs on the same side have overlapping reachable areas. This makes the gait analysis more complicated. One way to solve this problem is to eliminate all the overlapping reachable area, so that each leg has a distinct region which can be accessed only by itself, and not by any other leg. Furthermore, a rectangular region of the reachable area is defined, as shown in Fig. 2. Another way to solve this problem is to mark the region of the overlapping area so that the leg can reach those areas whenever interference does not occur [10]. Fig. 3 shows the model with markings of an overlapping area. By using this model, the robot can move with a longer stride length.

Let $P$ and $Q$ denote the size of a cell. Also, let $W$ be the distance between the reachable cell and the robot body, and $U$, the width of the 


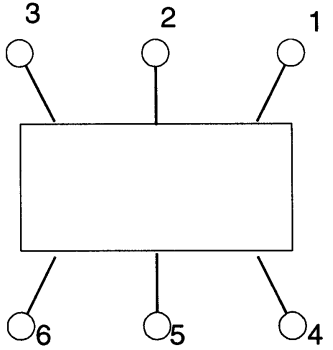

Fig. 1. Simplified hexapod model.

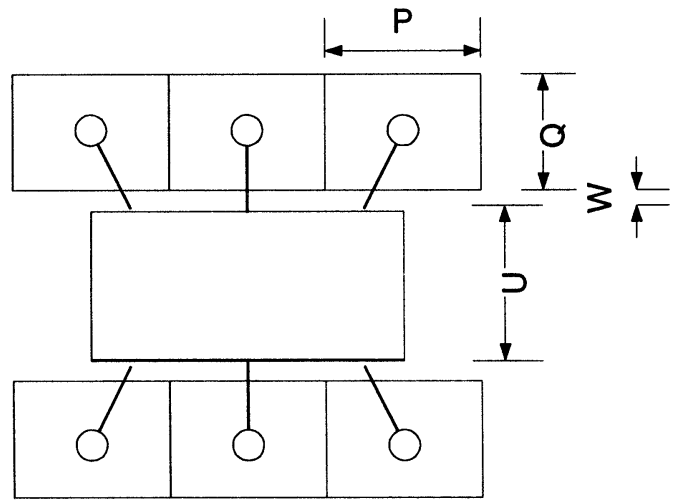

Fig. 2. Model with rectangular reachable area.

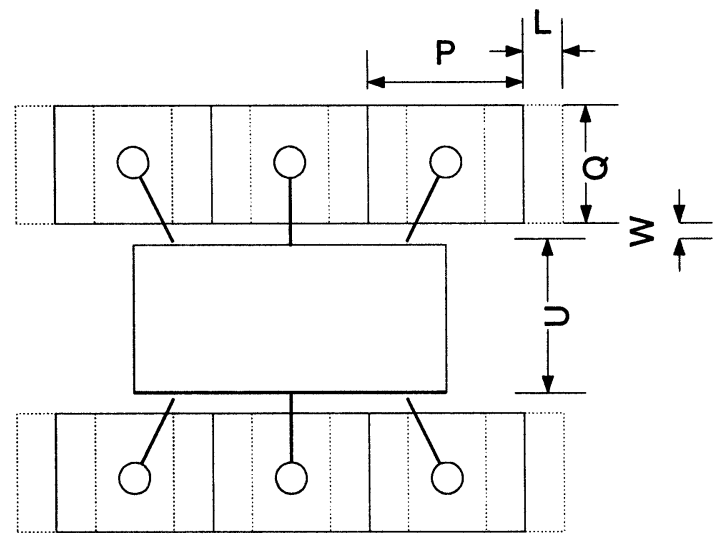

Fig. 3. Model with extended reachable area.

robot body. For the second model, $P^{\prime}=P+2 L$ is the length of the extended reachable area, where $L$ is less than $0.5 P$. Yang and Kim [9] have proposed a fault-tolerance gait for the hexapod robot based on this model. Assumptions are made for the simplicity of analysis. They include the following.

1) The hexapod has a symmetric structure.

2) The contact between a foot and the ground is a point.

3) There is no slipping between the foot and the ground.

4) All the mass of the legs is lumped into the body, and the center of gravity is assumed to be at the centroid of the body.

5) The initial foothold positions should be at the specified locations before the locomotion starts.

6) The speed of the hexapod body when it moves and the average speed of each leg during the transfer phase are constant.

They have defined a fault stability margin $S_{f}$, which is the minimum of stability margins of gaits, generated by changing alternately the state of one supporting leg of the gait to transfer state, and maintaining the other legs' states. Fault tolerance gait is formed so that, $S_{f}$ is non-negative during a whole locomotion.
Another useful term in gait analysis is stride length. It is defined as the distance which the center of gravity translates during one complete locomotion. Fault is defined as failure in the kinematic part of a leg and failure of communication between controller and a leg effector. The fault occurrence situations from [9] are 1) only one fault event occurs during a whole locomotion; and 2) the fault is not recovered during the locomotion.

\section{HeXagonal Model}

Inspired by the work of Yang and Kim [9], we also focus on the fault tolerance gait design, but on a different model. The main difference between the hexagonal model and the rectangular one, is that the former one makes use of an equilateral hexagon as the robot body. One leg is attached to each side of the hexagon. Fig. 4 gives the relationship between the reachable aread and annulus, and Fig. 5 shows the model. To make referral easier, legs are numbered anticlockwise starting from the bottom, as shown in Fig. 5. Comparison assumes the same leg design of the robot would be employed for two models. That means each leg has a reachable area in the form of an annulus and $r_{\max }$ is the radius of the annulus.

For the rectangular model, the area occupied by the robot body is equal to $2 P \times U$. For the hexagonal model, the body area is $\left(l^{2} \sin 60^{\circ} / 2\right) \times 6$. Practically, the body area of the model should be equal, for better comparison since most of the electronic and mechanical parts are placed on the top of the body. This is a minor point, however, because the size of the electronic and mechanical parts depend upon many factors, such as, the packaging of the electronic components.

It is reasonable to assume $U=P$. By using this assumption, we can get the area of the robot body $2 P^{2}$. Then the relationship between $P$ and $l$ is given by

$$
\begin{aligned}
2 P^{2} & =l^{2} \frac{3 \sqrt{3}}{2} \\
P^{2} & =l^{2} \frac{3 \sqrt{3}}{4} \\
\text { approximately } P & =1.1398 l \text { or } l=0.8774 P .
\end{aligned}
$$

A more important constraint for comparison is the size of the reachable area. As stated in [9], $P$ and $Q$ with $r_{\max }$ and $r_{\min }$ is shown graphically in Fig. 4. Mathematically

$$
r_{\max }^{2}=\left(r_{\min }+Q\right)^{2}+\left(\frac{1}{2} P\right)^{2} .
$$

\section{A. Fault-Tolerant Gait}

Fig. 6 shows the leg placing sequence of the fault-tolerant gait. The hexapod is moving in the positive $x$-direction. The number at the left upper corner shows the step number. The black dot represents the foothold position of the leg in supporting phase. The yellow dot indicates the leg in the swing phase. The red dot represents the center of gravity of the robot. Each leg is only restricted to move within the reachable area. The blue lines in each model illustrate the area enclosed by the supporting legs. The further away the center of gravity is from these blue lines, the more stable the robot is. Initially, the foothold of all the legs can be placed at the middle of the annulus, with $1 / 2 r_{\max }$ from the center point of the sector and equal distance from both sides of the sector. The hexapod will travel in periodic quadruped gaits so that $S_{f} \geq 0$. With this kind of fault-tolerant gait, the stride length of the robot can achieve $1 / 2 r_{\max }$. To prove this, we need to verify whether each leg can achieve $1 / 2 r_{\max }$. Since legs are moved in pairs (leg $1 \& 4$, leg $2 \& 5$, leg $3 \& 6$ ), verification can only apply to these combinations. Obviously, leg $1 \& 4$ can achieve $1 / 2 r_{\max }$, it 


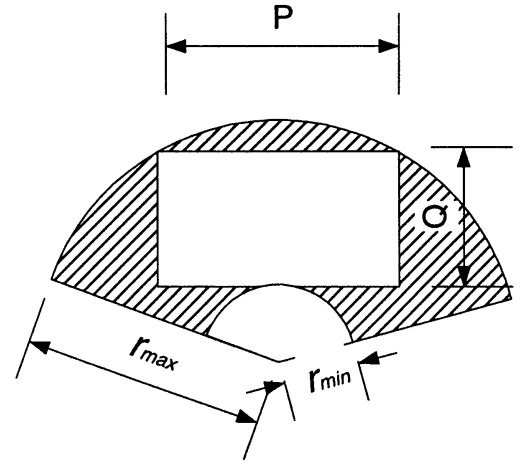

Fig. 4. Relationship between the reachable area and annulus.
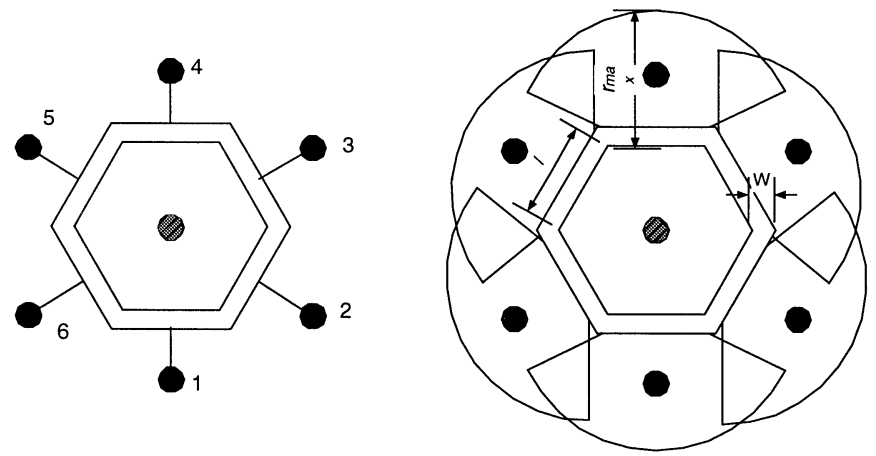

Fig. 5. Simplified hexagon mode.

remains leg pairs ( $2 \& 5)$ and ( $3 \& 6)$. Moreover, leg pairs $(2 \& 5)$ and $(3 \& 6)$ are symmetric pairs, so only one of those needs to be verified. Without loss of generality, leg 6 is chosen for verification. For leg 6 to move in positive $x$-direction, using simple geometrical calculation, we can prove leg 6 can move $0.65 r_{\max }$ until it reaches the boundary of the annulus.

\section{B. Stride Length}

It should be noted that the maximum stride length for the rectangular model for fault-tolerant locomotion is equal to $0.5 P$. For the hexagonal model, using a fault-tolerance gait, i.e., with the center of gravity falls at the cross-point of the supporting legs, the maximum stride length is equal to $0.5 r_{\max }$. For $r_{\max }>P$.

To obtain a greater stride length for a hexagonal model, it is desired that $r_{\max }>P$. Hence, from (2)

$$
\begin{aligned}
\left(r_{\min }+Q\right)^{2}+\left(\frac{1}{2} P\right)^{2} & >P^{2} \\
\left(r_{\min }+Q\right)^{2} & >\left(\frac{3}{4} P^{2}\right) .
\end{aligned}
$$

Let $r_{\min }$ be zero, which implies that $W=0 . W$ is the distance between the reachable area and the robot body. $W=0$ means the hexapod has a kinematics structure just located outside the robot body. If $W<0$, it means the kinematics structure is located below the robot body.

By letting $r_{\min }$ equals to zero, we obtain the result:

$$
\begin{aligned}
\left(r_{\min }+Q\right) & >\left(\frac{\sqrt{3}}{2} P\right) \\
Q & >0.866 P .
\end{aligned}
$$

Hence, if the $Q / P$ ratio is greater than 0.866 , the hexagonal model has a greater stride length than rectangular one. Another advantage of the hexagonal model is that it is easier to change the direction of

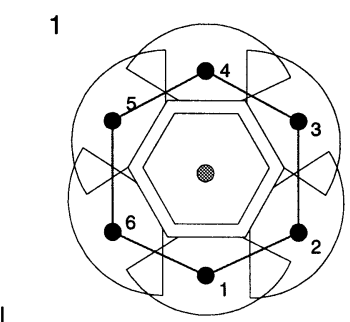

Initial foothold

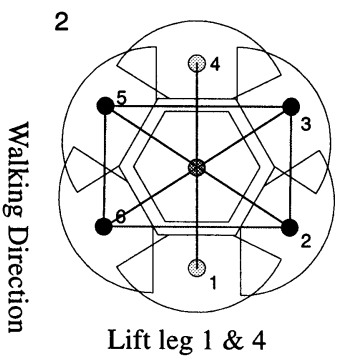

3
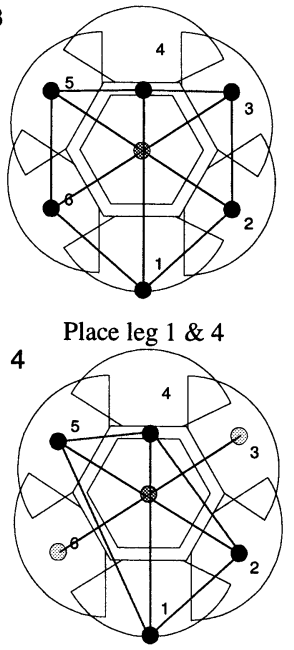

Lift leg $3 \& 6$

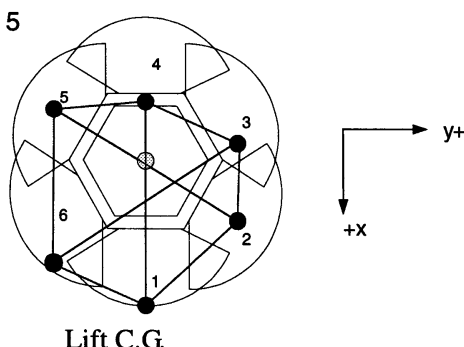

6

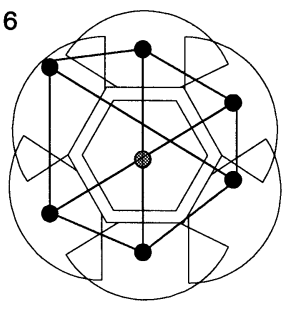

Move C.G.

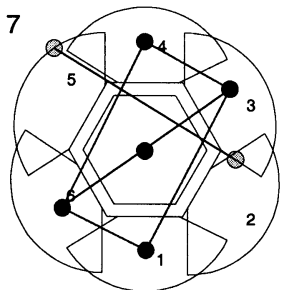

Lift leg 2 \& 5

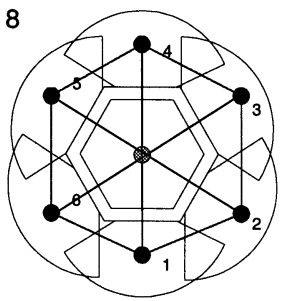

Place leg $2 \& 5$
Fig. 6. Fault tolerant gait sequence of hexagonal model.

movement. In the rectangular model case, crab walking is needed if the robot makes locomotion different from the longitudinal axis of the robot body. For the hexagonal model, by symmetry, however, there are already three longitudinal axes, and they can move in every 60 degree angle. Each pair of legs only needs to be responsible for 60-degree range of movement. This provides a simpler way for motion control.

\section{Turning Ability}

Turning of the hexapod depends on the location of the turning center [16]. In this paper, comparison is made on the case that the turning center is at the center of gravity. We assume that the tips of the legs are still at the center of the reachable areas after the turning, and we compare the maximum turning angle in one step. Fig. 7(a) shows the changes in foothold when the robot makes a turn of angle $\phi$ for the rectangular model. The distance and direction moved by each leg are different. The positional changes can be expressed in vector form. Let the position vector of the center of gravity be $C\left(C_{X}, C_{Y}\right)$, the position vector of each leg's center be $L C_{i}\left(L C i_{X}, L C i_{Y}\right)$ and the rotational matrix be $R(\phi)$. Then changes of foothold in vector form is expressed as

$$
\left[\left(L \vec{C}_{i}\right)-(\vec{C})_{\mid}\right] *(1-R \overrightarrow{(\phi)}) .
$$




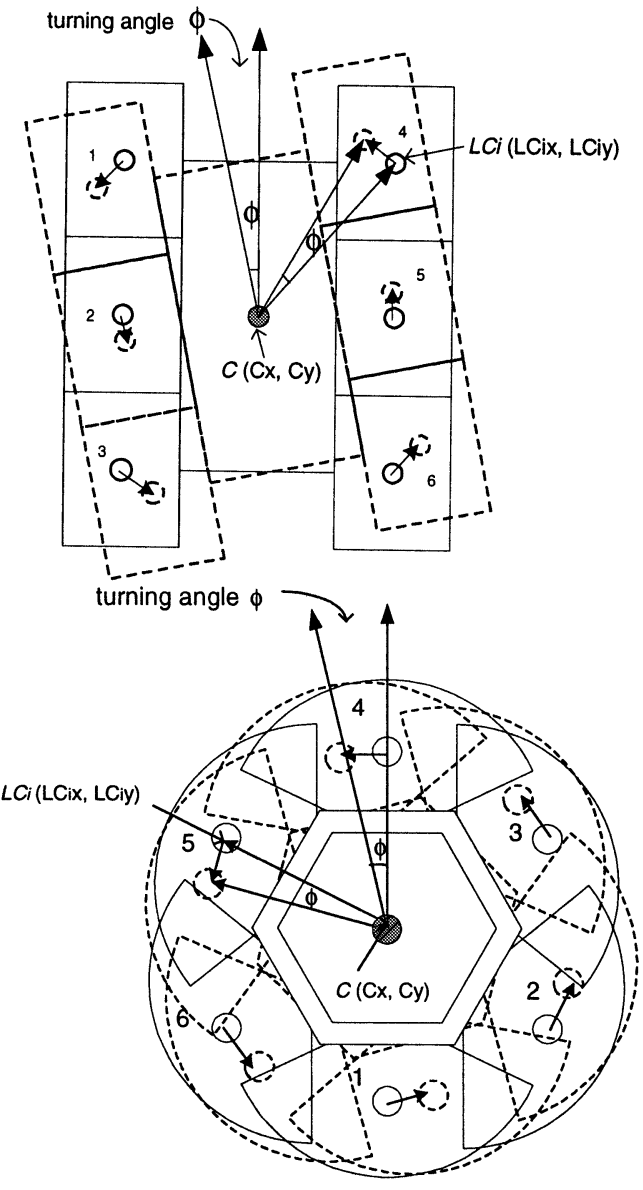

Fig. 7. (a) Turning step for rectangular model. (b) Turning step for hexagonal model.

As the robot moves in fault tolerant gait, the leg pairs moving sequences are $(3 \& 4),(2 \& 5)$, and $(1 \& 6)$. Since the turning center is at the center of gravity, the fault stability is always non-negative.

From Fig. 7(a), there is limitation on the turning angle of the rectangular model. The robot should make a turn such that the next foothold is within the reachable area. By graphical inspection, the condition for the robot to make a turn without excessing the reachable area is

$$
\begin{aligned}
& \frac{Q}{2 \cos \left(\frac{\pi-\phi}{2}-\tan ^{-1} \frac{2 P}{U+Q}\right)} \\
& >2 \sqrt{P^{2}+\left(\frac{1}{2}(Q+U)\right)^{2}} \sin \frac{\phi}{2} .
\end{aligned}
$$

This condition shows that the turning angle is related to the $Q, P$, and $U$. With the assumption that $U=P$, the changes in turning angle with $Q / P$ ratio is shown in Fig. 8. For the case $Q=0.866 P$, the turning angle is $\pm 2.27^{\circ}$. When $Q=P$, the turning angle is $\pm 24.298^{\circ}$. The higher the $Q / P$ ratio is, the greater the turning angle.

For the hexagonal model, it is easier to make turning about the center of gravity. The distance and the angular change of the six legs are identical in hexagonal model, which is easy to implement in both hardware and software. The amount of turning angle depends upon the size of the annulus in Fig. 4. The turning angle must be greater than $\pm 30^{\circ}$ if there is an overlapping area between adjacent legs, as shown in Fig. 7(b). It can be observed, therefore, that $\pm 30^{\circ}$ turning angle can be easily achieved in hexagonal case, while the turning angle is still less than $30^{\circ}$ even when $Q / P$ ratio equal to three. This has shown that the hexagonal model has better turning ability than the rectangular model.

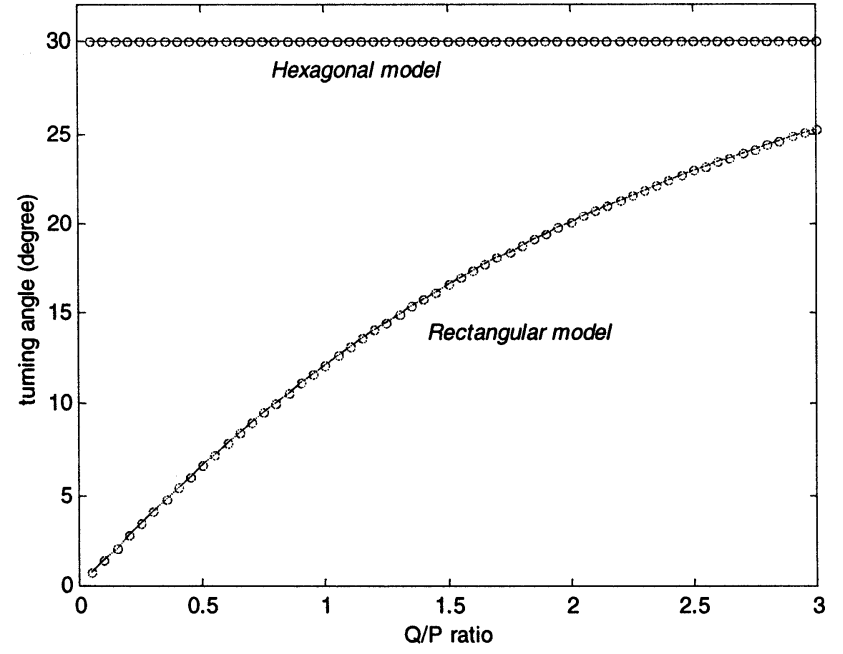

Fig. 8. Relationship between the turning angle and the $Q / P$ ratio.

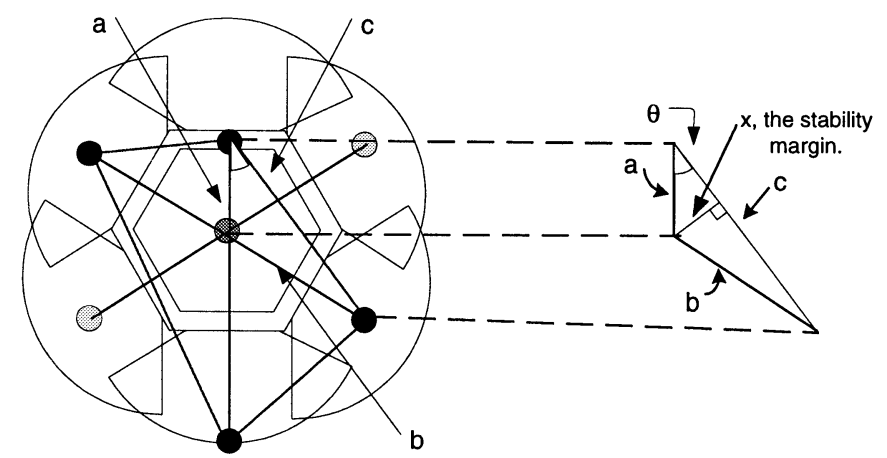

Fig. 9. Stability margin in one of the fault-tolerant gait.

\section{Stability Margin}

Fig. 6 shows the leg placing sequence of the fault-tolerance gait. The robot moves in a downward direction. From Fig. 6, the minimum distance between the center of gravity, and the boundary enclosed by the supporting legs, can be calculated. Among those leg placing sequence diagrams, step 4 is the most critical condition. The stability margin $x$ in step 4 is shown in Fig. 9.

$$
\begin{aligned}
& a=l \sin 60^{\circ} \\
& b=l \sin 60^{\circ}+\frac{1}{2} r_{\max } .
\end{aligned}
$$

By cosine rule

$$
c=\sqrt{a^{2}+b^{2}-2 a b \cos 120^{\circ}} .
$$

By sine rule

$$
\frac{\sin 120^{\circ}}{c}=\frac{\sin \theta}{b} \Rightarrow \sin \theta=\frac{b}{c} \sin 120^{\circ} .
$$

Hence, the stability margin $x$ is as follows:

$$
x=a \sin \theta \Rightarrow x=\frac{a b}{c} \sin 120^{\circ} .
$$

Using (1) and (3), $l$ and $Q$ can be expressed in term $P$, and then $a, b$, and $c$ can be calculated. This also implies that the comparison is based on same-size robot of the same size. Finally, the stability margin $x$ can be found equal to $0.4692 P$. The values of $l, Q, r_{\max }, a, b, c$, and $x$ are as follows:

$$
\begin{aligned}
l=0.8774 P, & Q=0.8660 P, \quad r_{\max }=P \\
x & =0.4692 P, \quad a=0.7598 P, \quad b=1.2598 P \\
c & =1.7669 P .
\end{aligned}
$$




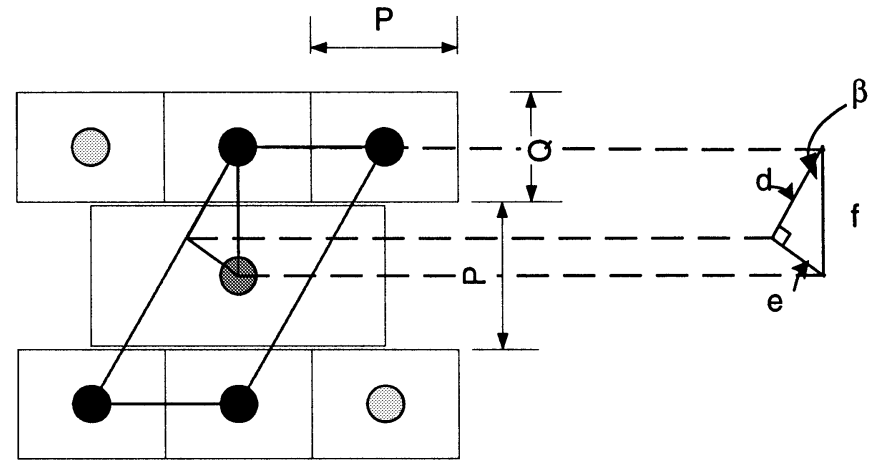

Fig. 10. Stability margin in one of the fault-tolerant gait of the rectangular model.

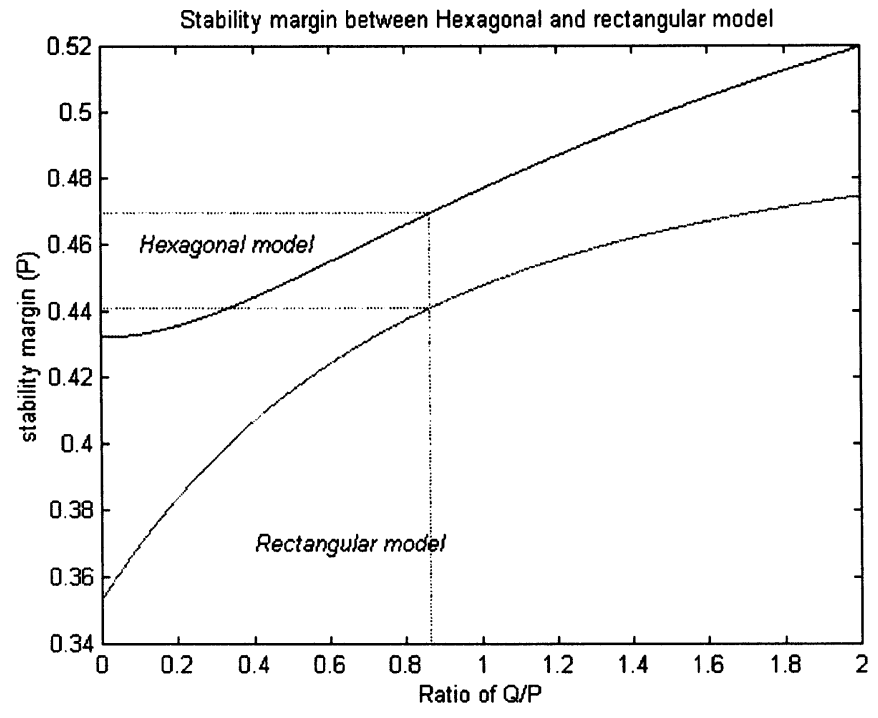

Fig. 11. Stability margin against ratio of $Q / P$.

Fig. 10 shows the smallest stability margin using fault tolerant gait in a rectangular model. Assume that $U=P$ and $Q=0.866 P$ (same stride length as in the hexagonal model), stability margin $e$ in this case is calculated.

$$
\tan \beta=\frac{P}{P+Q}
$$

Using (2)

$$
\begin{aligned}
Q & =0.866 P, \quad \beta=28.187^{\circ}, \quad e=f(\sin \beta) \\
f & =\frac{1}{2}(P+Q)=0.9330 P, \text { and } e=0.4407 P .
\end{aligned}
$$

From the above calculation, the hexagonal model with the same stride length shows a slightly better margin of stability. A more general relationship can be shown by plotting the stability margin of the two models against the ratio of $Q / P$. The result shows that the hexagonal model has higher stability margin for $Q / P$ ratio over the range from 0 to 1 . The dash lines in Fig. 11 indicate the case for $Q / P=0.8660$.

It should be noted that the upper limit of $e$ is $0.5 P$ for all positive $Q / P$. Also, it can be proved that $x$ is a monotonic increasing function for all positive values of $Q / P$. A more detail mathematical proof is included in the appendix. From the observation of Fig. 11, it can be seen that $x$ is already greater than $0.5 P$ when $Q / P>1.5$. Therefore, with increasing value of $x$, it must be greater than $e$ for all positive values of $Q / P$.

\section{CONCLUSIONS}

In this paper, we have made a number of comparisons between the hexagonal model and the rectangular model of hexapod robots. Assuming the same leg design and robot size, the hexagonal model shows better turning ability, higher stability margin and greater stride length in certain conditions. Mathematical relations between these two models have been formed. We hope this analysis can contribute to the hexapod design and development.

\section{REFERENCES}

[1] S. M. Song and K. J. Waldron, Machines That Walk: The Adaptive Suspension Vehicle. Cambridge, MA: MIT Press, 1989.

[2] D. J. Todd, Walking Machine: An Introduction to Legged Robots. London, U.K.: Kogan Page, 1985.

[3] R. D. Schraft, Service Robots: Products, Scenarios, Visions, A. K. Peters, Ed. Natick, MA, 2000.

[4] IEEE and Fraunhofer IPA Database on Service Robotics- Reconstruction Rosy. [Online]. Available: http://www.ipa.fhg.de/srdatabase/rosy.html

[5] J. Akizono et al., "Seabottom roughness measurement by aquatic walking robot," in Proc. MTS/IEEE Conf. OCEANS, vol. 2, 1997, pp. 1395-1398.

[6] Y. Tanaka and Y. Matoba, "Study of an intelligent hexapod walking robot," in IEEE/RSJ Intl. Workshop Intell. Rob. Syst. IROS, vol. 3, Nov. 1991, pp. 1535-1540.

[7] R. B. McGhee and G. I. Iswandhi, "Adaptive locomotion of a multilegged robot over rough terrain," IEEE Trans. Syst., Man, Cybern., vol. SMC-9, no. 4, pp. 176-182, 1979.

[8] A. P. Bessonov and N. V. Umnov, "The analysis of gaits in six-legged vehicles according to their static stability," in Proc. Symp. Theory Practice Rob. Manipulat., Udine, Italy, 1973.

[9] J. M. Yang and J. H. Kim, "Fault-tolerant locomotion of the hexapod robot," IEEE Trans. Syst., Man, Cybern., vol. 28, pp. 109-116, 1998.

[10] —, "Optimal fault tolerant gait sequence of the hexapod robot with overlapping reachable areas and crab walking," IEEE Trans. Syst. Man, Cybern., A, vol. 29, pp. 224-235, Mar. 1999.

[11] - "A fault tolerant gait for a hexapod robot over uneven terrain," IEEE Trans. Syst., Man, Cybern., B, vol. 30, pp. 172-180, Feb. 2000.

[12] Y. Baudoin and P. Alexandre, "Development of legged robots for use in disordered environments," in Proc. SPIE: Mobile Robots X, vol. 2591, Philadelphia, PA, 1995, pp. 100-106.

[13] Walking Robot Katharina. [Online]. Available: http://www1.iff.fhg.de/ iff/aut/english/start_katharina_e.html

[14] T. G. Bartholet, "The first functionoid developed by ODETICS inc," in Proc. Int. Conf. Advanc. Robot., 1983, pp. 293-298.

[15] A. Preumont, P. Alexandre, and D. Ghuys, "Gait analysis and implementation of a six leg walking machine," in Proc. Fifth Int. Conf. Advanc. Robot., vol. 2, 1991, pp. 941-945.

[16] C. D. Zhang and S. M. Song, "Turning gait of a quadrupedal walking machine," in Proc. IEEE Int. Conf. Robot. Automat., vol. 3, 1991, pp. 2106-2112. 